OPEN

SUBJECT AREAS:

QUANTUM INFORMATION

QUBITS

Received

27 June 2014

Accepted

26 August 2014

Published

18 September 2014

Correspondence and requests for materials should be addressed to G.-M.Z. Igmzeng@jlu. edu.cn) or L.-A.W.

(lianao_wu@ehu.es)

\title{
Symmetry restoration and quantumness reestablishment
}

\author{
Guo-Mo Zeng' , Lian-Ao Wu $u^{2,3} \&$ Hai-Jun Xing'
}

\begin{abstract}
${ }^{1}$ College of Physics, Jilin University, Changchun 130012, China, ${ }^{2}$ Department of Theoretical Physics and History of Science, The Basque Country University (EHU/UPV), PO Box 644, 48080 Bilbao Spain, ${ }^{3}$ Ikerbasque, Basque Foundation for Science, 48011 Bilbao Spain.
\end{abstract}

A realistic quantum many-body system, characterized by a generic microscopic Hamiltonian, is accessible only through approximation methods. The mean field theories, as the simplest practices of approximation methods, commonly serve as a powerful tool, but unfortunately often violate the symmetry of the Hamiltonian. The conventional BCS theory, as an excellent mean field approach, violates the particle number conservation and completely erases quantumness characterized by concurrence and quantum discord between different modes. We restore the symmetry by using the projected BCS theory and the exact numerical solution and find that the lost quantumness is synchronously reestablished. We show that while entanglement remains unchanged with the particle numbers, quantum discord behaves as an extensive quantity with respect to the system size. Surprisingly, discord is hardly dependent on the interaction strengths. The new feature of discord offers promising applications in modern quantum technologies.

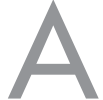

quantum many-body Hamiltonian $H$ often possesses invariance under symmetry operations, exemplified by the particle number $N$ and the angular momentum $J^{2}, J_{z}$. Proper treatments of symmetry are of great importance in developing approximation methods for the Hamiltonian, in particular for strongly correlated systems. Unfortunately, ubiquitous approximation methods such as the mean field approach usually require breaking the symmetry of the Hamiltonian. For instance, the renowned BCS theory ${ }^{1}$, proposed for superconductivity and later for nuclear system, employs simple product wave functions of independent quasi-particles to reveal the underlying physics, at a price of violating the particle-number conservation. The entire system undergoes a phase transition to a symmetry-violating superfluid phase. In condensed matter physics, the particlenumber fluctuation of a bulk superconductor is weak and the symmetry breaking does not play a significant role. On the contrary, the violation manifests itself in finite-size ensembles such as superconductive grains ${ }^{2}$ and nuclear systems. For instance, the nuclear properties obtained in the BCS treatment are the average of the target nucleus and its adjacent nuclei. The BCS theory successfully captures the dominant quantum correlation, i.e. the pairing effect between single particle (electron or nucleon) states and their time-reversals, but thoroughly washes out other correlations such as the correlations between different modes. These correlations might not be important for bulk superconductors but apparently exist in finite-size systems, and can be restored by the symmetryrestoration methods that go beyond the mean field approaches.

In quantum information theory, quantum and classical correlations can be distinguished specifically. Quantum correlations characterize quantumness, measured by concurrence and quantum discord, of a correlated system. It should be of great interest how quantumness varies with the process from violation to restoration of symmetry. Here we study a two-level BCS model. The conventional BCS theory of the model violates the particlenumber conservation. We shall restore the conservation by the projected BCS (PBCS) method, which projects out states with fixed particle numbers from the BCS wave function. It is noticeable that the lost quantumness is found when the symmetry is restored. Quantumness and symmetry are destroyed and reestablished simultaneously. We study the finite particle number effects on both concurrence and quantum discord in the case of large degeneracy. To our surprise, while entanglement drops greatly with the particle number from the maximum at two end points and then remains at half value, quantum discord behaves as an extensive quantity for a target system with a fixed degeneracy. This implies that the paring interaction is effectively transparent to discord. Physically, discord displays quantumness where a pair of qubits moves independently as if other qubits do not exist, whereas the existence of other qubits severely affects the entanglement. According to their definitions, quantum discord is the quantumness associated with quantum measurement, e.g. von Neumann measurement, on a system, while entanglement is from the density matrix itself of the system. The difference is crystal clear. Quantum discord seems to be a more general and fundamental nonclassical correlation than entanglement as pointed out in recent 
literatures ${ }^{3-6}$. As proved in Ref. 6, the set of states with zero-discord has volume zero in the whole Hilbert space, very different from the set of non-entangled states which have finite volumes. Since almost all quantum states may be useful resources for discord-dependent tasks due to their positive discord, our result that discord acts as an extensive quantity, can be important in assessing availability of a quantum state in various quantum information tasks.

The BCS theory ${ }^{1}$ was proposed to determine the fully paired ground state of superconductors and later was translated into the theories of nuclear structure ${ }^{7}$ since the pairing correlation was found in low-lying states of even-even nuclei. In the past decade some authors studied the entanglement of superconductors in the frame of BCS model considering that it provides a solution to a quantum many-body problem with an explicit wave function. Ref. 8 used concurrence to quantify the entanglement of the BCS state of superconducting compounds. Ref. 9 analyzed the finite-size properties of the two-level BCS model and discussed the entanglement properties of the ground state via concurrence. Without loss of generality, we now focus on the BCS application in nuclear systems, however, the conclusions obtained can be applied to any finite systems directly.

The ground-state BCS wave function of an even-even nucleus reads ${ }^{10}$

$$
|\mathrm{BCS}\rangle=\prod_{k>0}\left(u_{k}+v_{k} a_{k}^{\dagger} a_{\bar{k}}^{\dagger}\right)|0\rangle
$$

which violates particle-number conservation. Here $a_{k}^{\dagger}$ and $a_{\bar{k}}^{\dagger}$ are fermionic creation operators of single particle state $k$ and its timereversal $\bar{k}$, respectively. $u_{k}$ and $v_{k}$ are real variational parameters satisfying the normalization condition $u_{k}^{2}+v_{k}^{2}=1$. These parameters are determined by variation of the energy, with the restrictions that the expectation value of particle number operator $\hat{N}$ is equal to the practical particle number $N$, i.e., $\langle\mathrm{BCS}|\hat{N}| \mathrm{BCS}\rangle=N$.

The pure pairing interaction is widely used in nuclear BCS theory, for it provides a simple and powerful description of paring correlations in nuclei. A generic Hamiltonian with the pairing interaction reads

$$
H=\sum_{k>0} \epsilon_{k}\left(a_{k}^{\dagger} a_{k}+a_{\bar{k}}^{\dagger} a_{\bar{k}}\right)-\sum_{k, k^{\prime}>0} G_{k k^{\prime} k k^{\prime}} a_{k}^{\dagger} a_{\bar{k}}^{\dagger} a_{\bar{k}^{\prime}} a_{k^{\prime}}
$$

where $\epsilon_{k}{ }^{\prime}$ s are single-particle energies and $G_{k k^{\prime} k k^{\prime}}$ are the strengths of paring force dependent on the energy levels. The BCS theory is convenient and widely used. However it violates the particle number conservation and the physics obtained from BCS treatment is an average of the target nucleus and its adjacent nuclei. This symmetry breaking can be restored by the PBCS theory ${ }^{11}$, as introduced in Method.

We now construct three bilinear fermionic operators, $S_{+}^{(k)}=a_{k}^{\dagger} a_{\bar{k}}^{\dagger}$, $S_{-}^{(k)}=a_{\bar{k}} a_{k}$ and $S_{0}^{(k)}=\frac{1}{2}\left(a_{k}^{\dagger} a_{k}+a_{\bar{k}}^{\dagger} a_{\bar{k}}-1\right)$, which play the same role as spin operators (half of Pauli operators) and generate an $s u(2)$ algebra. The operator $S_{0}$ has eigenvalues $\pm \frac{1}{2}$ depending on whether the pair state $(k, \bar{k})$ is occupied or not. We denote the empty and occupied with $|0\rangle$ and $|1\rangle$, two states supporting a qubit. The equivalence between fermonic pairs and qubits is discussed in Refs. 1214. Specifically, a fermonic pair in $(k, \bar{k})$ corresponds to the $k$ th qubit.

\section{Results}

Consider a simplified pairing Hamiltonian (2) (similar to the nuclear model in Ref. 15, 16): two degenerate single particle energy levels, set as $\epsilon_{1}=0$ and $\epsilon_{2}=1$, with the degeneracies $\Omega_{1}$ and $\Omega_{2}$, respectively. The strengths of paring force are $G_{k \bar{k} k^{\prime} k^{\prime}}=4 G_{i i}(i=1,2)$ for both $k$ and $k^{\prime}$ in the same level $i$ and $G_{k \bar{k} k^{\prime} \bar{k}^{\prime}}=4 G_{12}=4 G_{21}$ for $k$ and $k^{\prime}$ in different levels. We focus on the low-lying states where all particles are coupled in pairs. The BCS and PBCS wave functions are given by Eqs. (1) and (32) in Methods.

Eq. (1) can be rewritten such that the BCS wave function becomes a direct product state of qubit states of different modes. Both concurrence and quantum discord are therefore vanishing. It shows that while violating particle number conservation, BCS wave function annihilates quantumness of different modes.

Now we calculate the reduced density matrix of two qubits $A$ and $B$ under the PBCS wave function ${ }^{10}$. The Hilbert space of the two qubits is spanned by the computational base, $\{|00\rangle,|01\rangle,|10\rangle,|11\rangle\}$, where in each basis, the first (second) digit indicates the state of qubit $A(B)$. There are three different types of reduced density matrices, determined by the way qubits $A$ and $B$ occupy the two energy levels, and all of them are a particular case of two-qubit $X$ states $^{17-20,22-24}$ with $\rho_{14}=$ $\rho_{41}=0$, which in general reads

$$
\rho=\left(\begin{array}{cccc}
\rho_{11} & 0 & 0 & 0 \\
0 & \rho_{22} & \rho_{23} & 0 \\
0 & \rho_{32} & \rho_{33} & 0 \\
0 & 0 & 0 & \rho_{44}
\end{array}\right),
$$

We distinguish the three types by superscripts (1), (2) and (3). Type (1) denotes the case that both qubits $A$ and $B$ are in the lower level; (2) $\mathrm{A}$ and $\mathrm{B}$ in the lower and the higher levels, respectively; (3) both $A$ and $B$ in the higher level. The non-zero matrix elements of $\rho^{(1)}$ are:

$$
\begin{aligned}
\rho_{11}^{(1)} & =C \sum_{i=\max \left\{0, p-\Omega_{2}\right\}}^{\min \left\{p, \Omega_{1}-2\right\}}\left(\begin{array}{c}
\Omega_{1}-2 \\
i
\end{array}\right)\left(\frac{v_{1}^{2}}{u_{1}^{2}}\right)^{i}\left(\begin{array}{c}
\Omega_{2} \\
p-i
\end{array}\right)\left(\frac{v_{2}^{2}}{u_{2}^{2}}\right)^{p-i}, \\
\rho_{22}^{(1)} & =\rho_{33}^{(1)}=\rho_{23}^{(1)}=\rho_{32}^{(1)} \\
& =C \sum_{i=\max \left\{1, p-\Omega_{2}\right\}}^{\min \left\{p, \Omega_{1}-1\right\}}\left(\begin{array}{c}
\Omega_{1}-2 \\
i-1
\end{array}\right)\left(\frac{v_{1}^{2}}{u_{1}^{2}}\right)^{i}\left(\begin{array}{c}
\Omega_{2} \\
p-i
\end{array}\right)\left(\frac{v_{2}^{2}}{u_{2}^{2}}\right)^{p-i}, \\
\rho_{44}^{(1)} & =C \sum_{i=\max \left\{2, p-\Omega_{2}\right\}}^{\min \left\{p, \Omega_{1}\right\}}\left(\begin{array}{c}
\Omega_{1}-2 \\
i-2
\end{array}\right)\left(\frac{v_{1}^{2}}{u_{1}^{2}}\right)^{i}\left(\begin{array}{c}
\Omega_{2} \\
p-i
\end{array}\right)\left(\frac{v_{2}^{2}}{u_{2}^{2}}\right)^{p-i},
\end{aligned}
$$

where $\left(\begin{array}{l}n \\ k\end{array}\right)$ stands for the number of combination, the common factor $C=u_{1}^{2 \Omega_{1}} u_{2}^{2 \Omega_{2}}$. For the second type, the matrix elements are

$$
\begin{aligned}
& \rho_{11}^{(2)}=C \sum_{i=\max \left\{0, p-\Omega_{2}+1\right\}}^{\min \left\{p, \Omega_{1}-1\right\}}\left(\begin{array}{c}
\Omega_{1}-1 \\
i
\end{array}\right)\left(\frac{v_{1}^{2}}{u_{1}^{2}}\right)^{i}\left(\begin{array}{c}
\Omega_{2}-1 \\
p-i
\end{array}\right)\left(\frac{v_{2}^{2}}{u_{2}^{2}}\right)^{p-i}, \\
& \rho_{22}^{(2)}=C \sum_{i=\max \left\{0, p-\Omega_{2}\right\}}^{\min \left\{p-1, \Omega_{1}-1\right\}}\left(\begin{array}{c}
\Omega_{1}-1 \\
i
\end{array}\right)\left(\frac{v_{1}^{2}}{u_{1}^{2}}\right)^{i}\left(\begin{array}{c}
\Omega_{2}-1 \\
p-i-1
\end{array}\right)\left(\frac{v_{2}^{2}}{u_{2}^{2}}\right)^{p-i}, \\
& \rho_{33}^{(2)}=C \sum_{i=\max \left\{0, p-\Omega_{1}\right\}}^{\min \left\{p-1, \Omega_{2}-1\right\}}\left(\begin{array}{c}
\Omega_{1}-1 \\
p-i-1
\end{array}\right)\left(\frac{v_{1}^{2}}{u_{1}^{2}}\right)^{p-i}\left(\begin{array}{c}
\Omega_{2}-1 \\
i
\end{array}\right)\left(\frac{v_{2}^{2}}{u_{2}^{2}}\right)^{i},
\end{aligned}
$$$$
\rho_{23}^{(2)}=\rho_{32}^{(2)}=\sqrt{\rho_{22}^{(2)} \rho_{33}^{(2)}} \text {, }
$$$$
\rho_{44}^{(2)}=C \sum_{i=\max \left\{1, p-\Omega_{2}\right\}}^{\min \left\{p-1, \Omega_{1}\right\}}\left(\begin{array}{c}
\Omega_{1}-1 \\
i-1
\end{array}\right)\left(\frac{v_{1}^{2}}{u_{1}^{2}}\right)^{i}\left(\begin{array}{c}
\Omega_{2}-1 \\
p-i-1
\end{array}\right)\left(\frac{v_{2}^{2}}{u_{2}^{2}}\right)^{p-i} .
$$

The reduced density matrix of third type is similar to first one. When $\Omega_{1}=\Omega_{2}=\Omega$, the two types are connected by a simple relationship, $\rho^{(1)}(p)=\rho^{(3)}(2 \Omega-p)$, due to the particle-hole symmetry with $2 \Omega-p$ being the hole number.

For the X-type density matrix, one can derive the expression of concurrence as ${ }^{17}$

$$
\mathbb{C}(\rho)=\max \left\{0,2 \sqrt{\rho_{22} \rho_{33}}-2 \sqrt{\rho_{11} \rho_{44}}\right\} .
$$




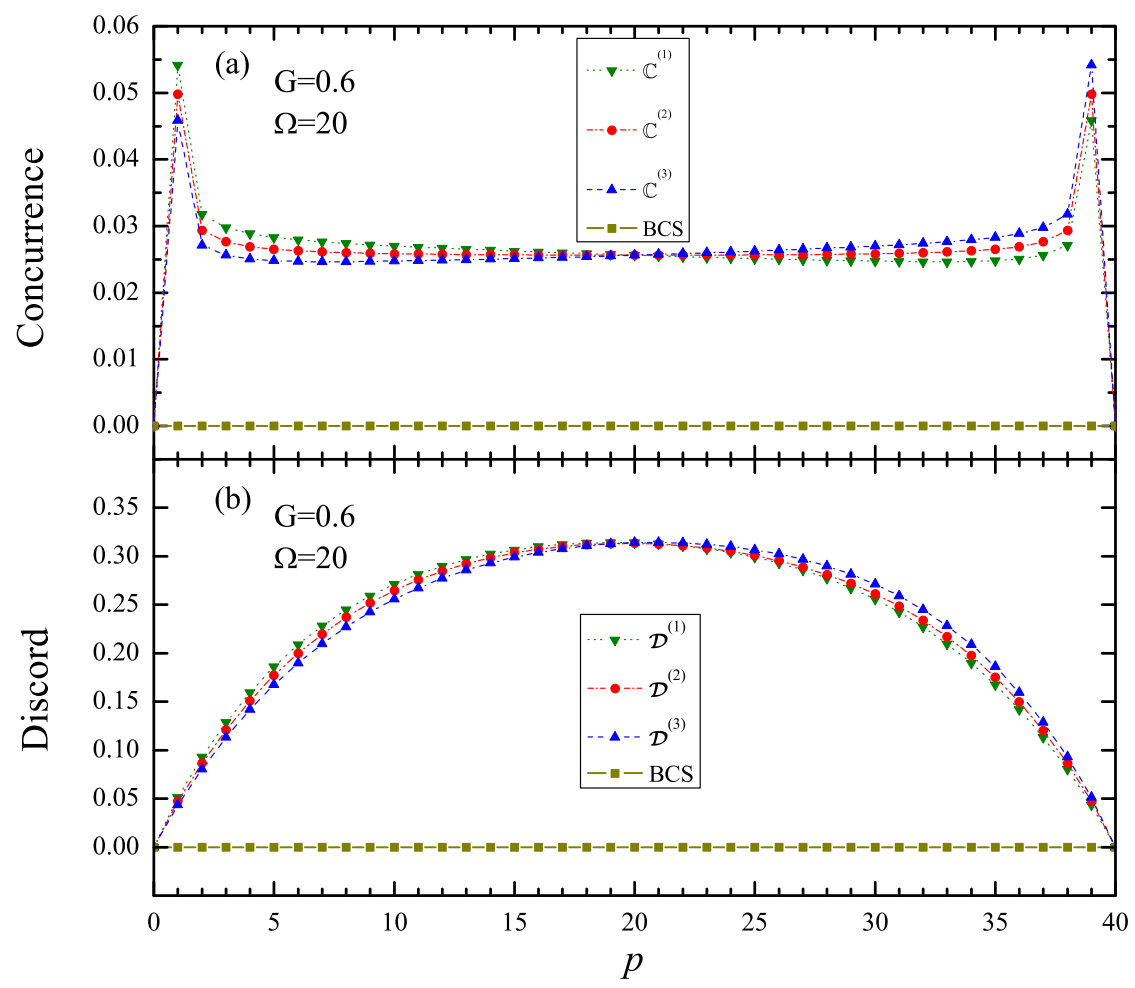

Figure $1 \mid$ PBCS calculations for (a) Concurrence and (b) Quantum discord versus particle-pair number $p$ at the paring strength $G=0.6$, single particle energies $\epsilon_{1}=0$ and $\epsilon_{2}=1$. Both levels have the same degeneracy $\Omega=20$. The results of BCS are also drawn as a reference. All connection lines between points are just for guiding eyes and the same below.

The total correlation of the system can be written as ${ }^{18}$

$$
\begin{aligned}
\mathcal{I}(\rho) & =\rho_{11} \log _{2} \rho_{11}+\rho_{44} \log _{2} \rho_{44}+\left(\rho_{22}+\rho_{33}\right) \log _{2}\left(\rho_{22}+\rho_{33}\right) \\
& -\left(\rho_{11}+\rho_{22}\right) \log _{2}\left(\rho_{11}+\rho_{22}\right)-\left(\rho_{33}+\rho_{44}\right) \log _{2}\left(\rho_{33}+\rho_{44}\right)(7) \\
& -\left(\rho_{11}+\rho_{33}\right) \log _{2}\left(\rho_{11}+\rho_{33}\right)-\left(\rho_{22}+\rho_{44}\right) \log _{2}\left(\rho_{22}+\rho_{44}\right) .
\end{aligned}
$$

The classical correlation is defined, based on the von Neumann measurements $\left\{B_{k}\right\}(k=0,1)$ on subsystem $B$, as

$$
\begin{aligned}
\mathcal{C}(\rho)= & -\left(\rho_{11}+\rho_{22}\right) \log _{2}\left(\rho_{11}+\rho_{22}\right) \\
& -\left(\rho_{33}+\rho_{44}\right) \log _{2}\left(\rho_{33}+\rho_{44}\right)-\min \left\{S_{1}, S_{2}\right\},
\end{aligned}
$$

where we follow the discussions of Ref. 20 and here

$$
\begin{aligned}
S_{1}= & -\rho_{11} \log _{2} \frac{\rho_{11}}{\rho_{11}+\rho_{33}}-\rho_{33} \log _{2} \frac{\rho_{33}}{\rho_{11}+\rho_{33}} \\
& -\rho_{22} \log _{2} \frac{\rho_{22}}{\rho_{22}+\rho_{44}}-\rho_{44} \log _{2} \frac{\rho_{44}}{\rho_{22}+\rho_{44}}
\end{aligned}
$$

and

$$
S_{2}=-\frac{1-\theta}{2} \log _{2} \frac{1-\theta}{2}-\frac{1+\theta}{2} \log _{2} \frac{1+\theta}{2}
$$

with

$$
\theta=\sqrt{\left(\rho_{11}+\rho_{22}-\rho_{33}-\rho_{44}\right)^{2}+4 \rho_{22} \rho_{33}}
$$

Two-level case. We now consider a pair-correlated system with two degenerate levels. We first compute concurrence and quantum discord based on PBCS for a simple case that $\Omega_{1}=\Omega_{2}=\Omega, G_{11}=$ $G_{12}=G_{21}=G_{22}=G$. The concurrence and quantum discord of three types versus pairing number $p$ are shown in Fig. 1 (a) and (b), respectively. At first glance, we notice that both curves of concurrence and quantum discord exhibit symmetry about $p=\Omega$, perfectly for the second type, and approximately for the first and third types, as well as the particle-hole symmetry between first case and third case as mentioned above. Thus, in the following we only need to concentrate our discussion on the region $0 \leq p \leq \Omega$ and one of the three types, e.g., type 2 . The more meaningful and essential result displayed in Fig. 1 is that concurrence and discord exhibit totally different behaviors. From Fig. 1 (a) we can see that concurrence starts with zero at $p=0$ corresponding to the BCS ground state, jumps to the maximal value at $p=1$, then decreases by near half and keeps almost unchanged with particle number. Clearly, it shows that symmetry restoration results in the reestablishment of entanglement.

On the other hand, Fig. 1 (b) shows that quantum discord also starts with zero, indicating that the BCS state is not only unentangled but also classic-only correlated. The different behavior of discord occurs after $p=1$, it does not drop like concurrence but increases with pair numbers until its highest value as the total maximal occupation number of the two levels are half-filled. This fact implies that concurrence and discord are different aspects of quantumness, owing to their distinct nature. There have been some reports about physical phenomena where entanglement and discord behave differently. A typical example is that discord can indicate the KosterlitzThouless phase transition in the XXZ model, whereas concurrence cannot $^{18,25,26}$.

In Fig. 2 we plot together the curves of concurrence and discord, as well as the total correlation $\mathcal{I}(\rho)$ and classical correlation $\mathcal{C}(\rho)$ (refer to Ref. 22 for detailed expressions) vs particle number for the second type with $\Omega_{1}=\Omega_{2}=20$. Apart from the remarkable difference between concurrence and quantum discord, we can also see that $\mathcal{D} \geq \mathcal{C}$ for the whole range of particle number, oppositing to the early speculation that $\mathcal{C} \geq \mathcal{D}$ for any quantum state ${ }^{27-29}$.

The Hamiltonian (2) can be exactly diagonalized. The numerical calculations show that the differences between PBCS and the exact solution will diminish with degeneracy and tend to vanish as the degeneracy is large enough. For a small degeneracy, e.g., $\Omega_{1}=\Omega_{2}$ 


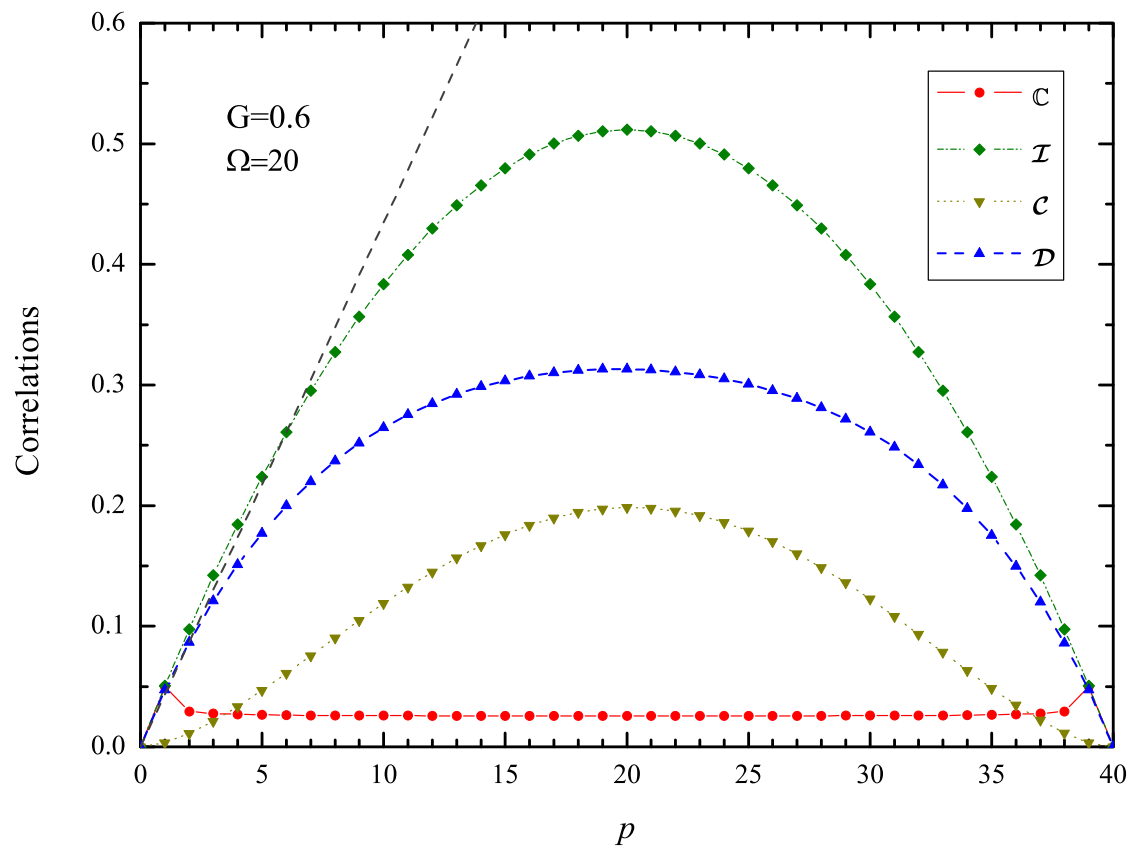

Figure $2 \mid$ Discord, concurrence, total correlation, and classical correlation versus pair number $p$ at the paring strength $G=0.6$, and $\Omega=20$. Single particle energies are $\epsilon_{1}=0$ and $\epsilon_{2}=1$.

$=6$, the fully restored quantum correlations measured by concurrence and discord in the exact solution are slightly larger than those in PBCS. It shows that PBCS is a perfect approximation to the exact solution in the sense of restoring the quantum correlations. However, superior to PBCS method, the exact diagonalization can even treat more complicated cases. In particular, we set $G_{11}=0.7, G_{12}=0.6, G_{22}=0.5$ for the fixed $\Omega_{1}=\Omega_{2}=6$, and $\Omega_{1}=7, \Omega_{2}=5$ for the fixed $G_{11}=G_{12}=G_{22}$ $=0.6$, respectively. Fig. 3 shows concurrence and quantum discord, and compare with a typical case that $\Omega_{1}=\Omega_{2}=6$ and $G_{11}=G_{12}=$ $G_{22}=0.6$. The deviations from the typical case are slight.

Comparing Fig. 2 and Fig. 3, we also notice that for larger degeneracy, quantum discord increases approximately linearly with particle (hole) pair, when $p, q \ll \Omega$, indicating that it acts as an extensive quantity like internal energy. We calculate the cases when $\Omega_{1} \neq \Omega_{2}$, and $G_{11} \neq G_{22}=G_{33} \neq G_{44}$, and confirm that these interesting properties remain the same. From Fig. 2, we can see that the quantum mutual information, i.e., the total correlation displays a much better linearity than discord. For $\mathrm{p} \leq 8$ the variation of total correlation versus $p$ is very close to a straight line with slope $\sim 0.42$. For $p \leq 4$ the quantum discord also coincides quite well with the straight line. An extensive quantity normally is the sum of the properties of separate noninteracting subsystems that compose the entire system. This implies astonishingly that the pairing interaction is effectively transparent to discord. For instance, discord as a function of the pairing strength $G: \mathcal{D}(G) \rightarrow 1.006(0.4), 1.003(0.5), 0.998(0.7)$, 0.997(0.8), $0.996(0.9)$ and $0.995(1.0)$ in unit of $\mathcal{D}(G=0.6)=0.03168$ when $\Omega$ $=120$ and $p=4$. The values of $\mathcal{D}(G)$ merely change with $G$, in particular when $G$ is strong. Physically, discord displays quantumness where a pair of qubits moves independently as if other qubits do not exist, whereas the existence of other qubits severely affects the values of entanglement. Based on their definitions, discord reflects the quantumness from quantum measurement on the interested system, meanwhile entanglement is from the density matrix directly of the system. In comparison with entanglement, discord quantifies more general and more fundamental quantum correlations $s^{3-6}$. In particular, Ref. 6 shows that the set of states with vanishing discord, i.e., the set of classical states, has volume zero in the whole Hilbert space. This is very different from the set of separable (non-entangled) states, which exhibits finite volume. This result provides another perspective for understanding the differences between entanglement and discord. Considering that the existence of discord determines the nontrivial properties of quantum states, it was shown that discord may be the resource responsible for the quantum speedup ${ }^{4}$ in computational models. This implies that almost all quantum states are useful resources due to their positive discord. The feature, discord as an extensive quantity, is expected to have new applications in quantum information practices.

One-level case. In order to understand the above results deeply, we consider a one-level system with degeneracy $\Omega$. This is the limit of the two-level cases when level difference vanishes. The density matrix now reads

$$
\rho=\frac{1}{\Omega(\Omega-1)}\left(\begin{array}{cccc}
q(q-1) & 0 & 0 & 0 \\
0 & p q & p q & 0 \\
0 & p q & p q & 0 \\
0 & 0 & 0 & p(p-1)
\end{array}\right)
$$

where $q=\Omega-p$ is the hole-pair number in the single level. The strength of paring force $G$ does not appear in the density matrix, which may be the reason why in the two-level case the entanglement and correlations are hardly affected by the strength of paring force. It turns out that it is paring correlation itself, rather than paring interaction, that determines these novel properties of a paircorrelated many-body system. Here we emphasize that $\rho$ is symmetric under the interchange of $p$ and $q$, or interexchange of states $|00\rangle$ and $|11\rangle$, so are the entanglement, classical correlations and quantum discord. In one-level case, all correlations have explicit analytical expressions, the concurrence is

$$
\mathbb{C}(\rho)=\frac{2}{\Omega(\Omega-1)}[p q-\sqrt{p(p-1) q(q-1)}],
$$

and the total correlation reads

$$
\begin{aligned}
\mathcal{I}(\rho)= & \frac{p(p-1)}{\Omega(\Omega-1)} \log _{2} \frac{p(p-1)}{\Omega(\Omega-1)}+\frac{q(q-1)}{\Omega(\Omega-1)} \log _{2} \frac{q(q-1)}{\Omega(\Omega-1)} \\
& +\frac{2 p q}{\Omega(\Omega-1)} \log _{2} \frac{2 p q}{\Omega(\Omega-1)}-\frac{2 p}{\Omega} \log _{2} \frac{p}{\Omega}-\frac{2 q}{\Omega} \log _{2} \frac{q}{\Omega},
\end{aligned}
$$




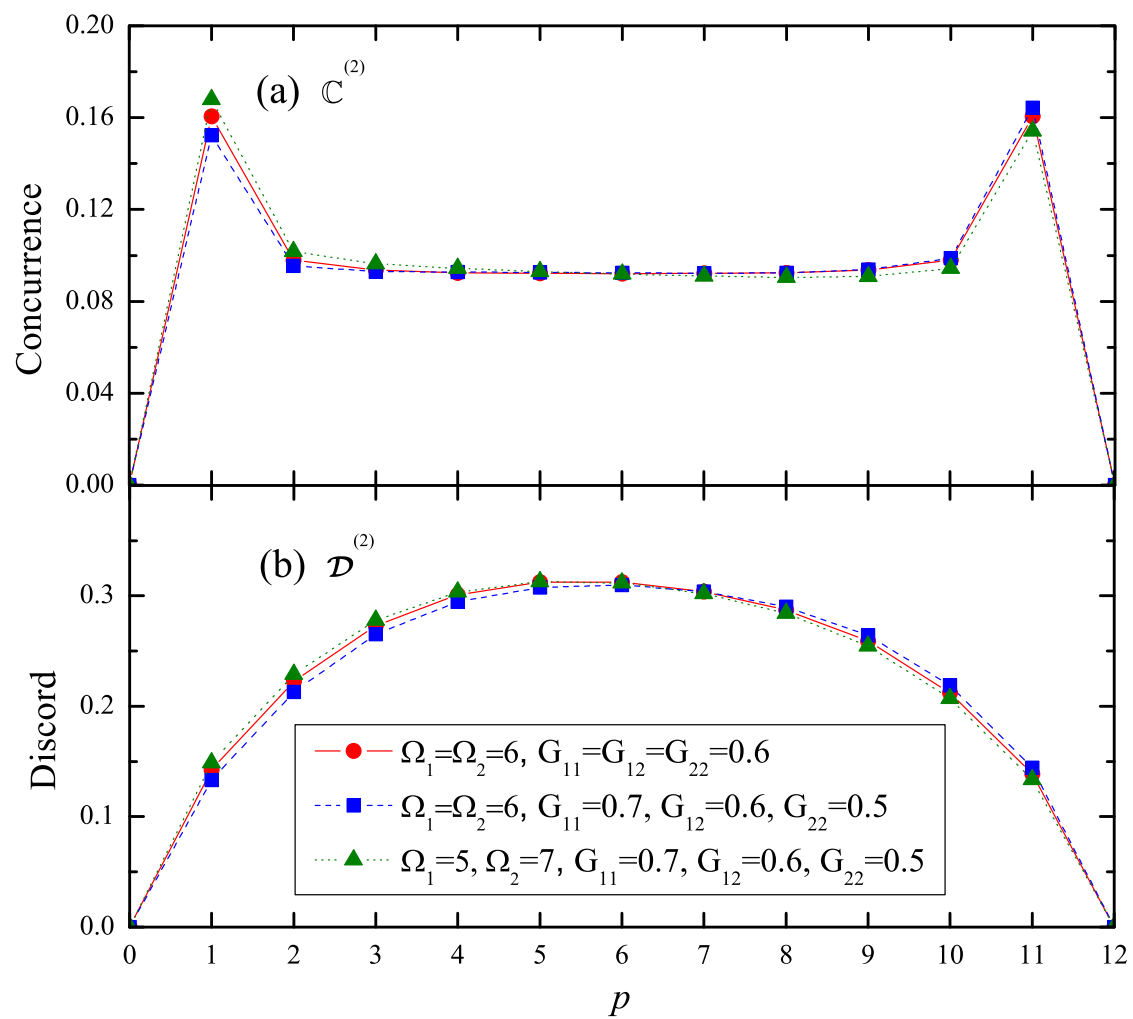

Figure 3 Concurrence and discord versus particle-pair number $p$ from the exact solutions of Hamiltonian (2). Single particle energies are $\epsilon_{1}=0$ and $\epsilon_{2}=1$.

As for classical correlation, we compare

$$
\begin{array}{r}
S_{1}=-\frac{p q}{\Omega(\Omega-1)} \log _{2} \frac{q}{\Omega-1}-\frac{p(p-1)}{\Omega(\Omega-1)} \log _{2} \frac{p-1}{\Omega-1} \\
-\frac{p q}{\Omega(\Omega-1)} \log _{2} \frac{p}{\Omega-1}-\frac{q(q-1)}{\Omega(\Omega-1)} \log _{2} \frac{q-1}{\Omega-1}
\end{array}
$$

and

$$
S_{2}=-\frac{1-\theta}{2} \log _{2} \frac{1-\theta}{2}-\frac{1+\theta}{2} \log _{2} \frac{1+\theta}{2}
$$

with

$$
\theta=\frac{1}{\Omega(\Omega-1)} \sqrt{(p-q)^{2}(\Omega-1)^{2}+4 p^{2} q^{2}}
$$

We find that $\min \left\{S_{1}, S_{2}\right\}=S_{2}$, thus the classical correlation is given by

$$
\begin{aligned}
\mathcal{C}(\rho)= & \frac{1-\theta}{2} \log _{2} \frac{1-\theta}{2}+\frac{1+\theta}{2} \log _{2} \frac{1+\theta}{2} \\
& -\frac{p}{\Omega} \log _{2} \frac{p}{\Omega}-\frac{q}{\Omega} \log _{2} \frac{q}{\Omega}
\end{aligned}
$$

and thereby the quantum discord is

$$
\begin{aligned}
\mathcal{D}(\rho)= & \frac{p(p-1)}{\Omega(\Omega-1)} \log _{2} \frac{p(p-1)}{\Omega(\Omega-1)}+\frac{q(q-1)}{\Omega(\Omega-1)} \log _{2} \frac{q(q-1)}{\Omega(\Omega-1)} \\
& +\frac{2 p q}{\Omega(\Omega-1)} \log _{2} \frac{2 p q}{\Omega(\Omega-1)}-\frac{p}{\Omega} \log _{2} \frac{p}{\Omega}-\frac{q}{\Omega} \log _{2} \frac{q}{\Omega} \\
& -\frac{1-\theta}{2} \log _{2} \frac{1-\theta}{2}-\frac{1+\theta}{2} \log _{2} \frac{1+\theta}{2} .
\end{aligned}
$$

These expressions are exactly symmetric under the interchange of $p$ and $q$, therefore all of them are even functions of $p-q$ and their extrema lie at $p=q=\Omega / 2$ and at the ends. In what follows we will focus our discussions on particle pair $p$ due to the symmetry. All results are the same once replacing $p$ with $q$. Concurrence $\mathbb{C}$ is a monotone decreasing function of $p$ for $p<\Omega / 2$ and reaches its minimum at $p=\Omega / 2$. However, for a fixed degeneracy $\Omega$, the values of concurrence hardly vary with $p$, as shown in the above equations. Concurrence has the limit, as $\Omega \gg 1, p \gg 1, p \ll \Omega$

$$
\mathbb{C}(\rho) \simeq \frac{1}{\Omega-1},
$$

which is the same as its minimum at $p=\frac{\Omega}{2}$. This indicates that concurrence behaves as an intensive quantity. On the other hand, concurrence vanishes at large $\Omega$ because of the factor $\frac{2}{\Omega(\Omega-1)}$ in Eq. (13). This result may be used to choose materials as the resource of quantumness according to their degeneracy and the number of particle (or hole) pairs.

On contrary to concurrence, the total correlation, classical correlation and quantum discord behave completely differently. They are monotonically increasing functions of $p$ in the intervals $p \in(0$, $\Omega / 2)$ with $p=\Omega / 2$ as the maximum. The maximal values are

$$
\begin{gathered}
\mathcal{I}_{\text {max }}=\log _{2} \frac{\Omega-2}{\Omega-1}+\frac{\Omega}{2(\Omega-1)} \log _{2} \frac{2 \Omega}{\Omega-2} \\
\mathcal{C}_{\max }=\frac{1}{4}\left(\frac{\Omega-2}{\Omega-1} \log _{2} \frac{\Omega-2}{\Omega-1}+\frac{3 \Omega-2}{\Omega-1} \log _{2} \frac{3 \Omega-2}{\Omega-1}\right)-1 \\
\mathcal{D}_{\text {max }}=\frac{1}{4}\left(\frac{3 \Omega-2}{\Omega-1} \log _{2} \frac{\Omega-2}{3 \Omega-2}+\frac{2 \Omega}{\Omega-1} \log _{2} \frac{2 \Omega}{\Omega-2}\right)+1
\end{gathered}
$$

When $\Omega \gg 1$, their limits are given by 


$$
\begin{gathered}
\mathcal{I}_{\max } \rightarrow \frac{1}{2}, \\
\mathcal{C}_{\max } \rightarrow \frac{3}{4} \log _{2} 3-1 \simeq 0.189, \\
\mathcal{D}_{\max } \rightarrow \frac{3}{2}-\frac{3}{4} \log _{2} 3 \simeq 0.311,
\end{gathered}
$$

It means that no matter how large the degeneracy is, the above correlations do not vanish but remain a constant.

Under the large degeneracy limits with small numbers of pairs, the total correlation, classical correlation and quantum discord are

$$
\begin{gathered}
\left.\mathcal{I}(\rho)\right|_{\Omega \gg 1, p \ll \Omega} \simeq \frac{2 p}{\Omega}, \\
\left.\mathcal{C}(\rho)\right|_{\Omega \gg 1, p \ll \Omega} \simeq-\frac{p}{\Omega} \log _{2} \frac{p}{\Omega}, \\
\left.\mathcal{D}(\rho)\right|_{\Omega \gg 1, p \ll \Omega} \simeq \frac{2 p}{\Omega}+\frac{p}{\Omega} \log _{2} \frac{p}{\Omega} .
\end{gathered}
$$

It is thus clear that the total correlation increases linearly with particle pair, while the quantum discord increases almost linearly, as observed in the two-level case where the slope is $\sim 0.42$ when $\Omega=40$. We therefore further confirm, by the analytical one-level case that the conclusions of discord being an extensive quantity in the two-level case remains valid and should imply profound universality for any many-body system consisting of pairwise correlated particles. These theoretical results are obviously testable experimentally. For example strong correlated many-body nuclear systems may allow to choose a series of even-even isotones, which usually have the same single particle levels for valence neutrons, to examine the dependance of quantum correlations on the number of paired neutrons. We can also select a group of nuclei which have the same number of valence protons (or neutrons) but different valence shells to check our results for quantum correlations.

\section{Discussion}

We have studied symmetry restoration and quantumness reestablishment of the BCS theory, as well as the relationship between paring correlation and quantumness in pair-correlated many-body systems. Restored entanglement may not matter for bulk superconductors because it keeps on a low level with particle-pair numbers. Quantum discord, on the other hand, is an extensive quantity and grows linearly with pair numbers. From the perspective of paring interaction, a pair of qubits moves independently, while surrounding qubits strongly affects entanglement of the target qubit pair. The underlying origin of these effects should be from that discord is a measurement-dependent quantity, while entanglement is only determined by the density matrix of the target system. Besides, because of the volume-zero of the set of classic states, these states can be hardly found in Hilbert space. Considering that in many physical systems, quantum discord behaves qualitatively similar to entanglement, it is significant to find physical entities where discord substantially differs from entanglement. An example is that discord signals KT phase transition while entanglement cannot. Our results display a new aspect of the differences, with respect to particle number effect, which may play a unique role in exploring strongly correlated systems like high temperature superconductors since the quantity is hardly dependent on interactions in strong interaction regime ${ }^{30}$. The new feature may hint profound physics behind quantum discord and may offer promising applications for, e.g., certain quantum computations.

It is worth mentioning that quantum discord can also be discussed in a more general setup of positive operator valued measurements
$(\mathrm{POVM})^{20,21}$. We can expect that, by considering POVM instead of von Neumann measurement, the main results will remain essentially the same. For X-states as considered here, extremization via POVM can be reduced to orthogonal projectors for a number of states ${ }^{21}$.

\section{Methods}

BCS and PBCS. By introducing the Lagrange multiplier $\lambda$, called the chemical potential, we determine the BCS parameters by variation of the BCS expectation value of $H^{\prime}=H-\lambda \hat{N}$

$$
\begin{aligned}
\left\langle\mathrm{BCS}\left|H^{\prime}\right| \mathrm{BCS}\right\rangle= & \sum_{k>0}\left(\epsilon_{k}-\lambda\right) v_{k}^{2}+\sum_{k, k^{\prime}>0} G_{k k^{\prime} k k^{\prime}} v_{k}^{2} v_{k^{\prime}}^{2} \\
& +\sum_{k, k^{\prime}>0} G_{k \bar{k} k^{\prime} \bar{k}^{\prime}} u_{k} v_{k} u_{k^{\prime}} v_{k^{\prime}},
\end{aligned}
$$

and yields two quadratic equations for $u_{k}^{2}$ and $v_{k}^{2}$,

$$
u_{k}^{2}=\frac{1}{2}\left(1-\frac{\tilde{\epsilon}_{k}}{\sqrt{\tilde{\epsilon}_{k}^{2}+\Delta_{k}^{2}}}\right), v_{k}^{2}=\frac{1}{2}\left(1+\frac{\tilde{\epsilon}_{k}}{\sqrt{\tilde{\epsilon}_{k}^{2}+\Delta_{k}^{2}}}\right),
$$

where $\tilde{\epsilon}_{k}=\epsilon_{k}+\sum_{k^{\prime}>0}\left(G_{k k^{\prime} k k^{\prime}}+G_{\bar{k} k^{\prime} k^{\prime}}\right) v_{k^{\prime}}^{2}-\lambda$ and the gap parameters

$\Delta_{k}=-\sum_{k^{\prime}>0} G_{k \bar{k} k^{\prime} k^{\prime}} u_{k^{\prime}} v_{k^{\prime}}$. The BCS method provides the convenience that we can treat a nucleus as a system of quasi-particles independently moving in a mean field. However it violates the particle number conservation.

This symmetry breaking can be restored by projection techniques, of which the method of residues ${ }^{11}$ employs a projector $\hat{P}^{A}=\frac{1}{2 \pi i} \oint \frac{z^{\hat{N}}}{z^{A+1}} d z$ to act on the BCS wave function Eq.(1)

$$
\left|\Psi^{N}\right\rangle=\hat{P}^{N=2 p}|\Phi\rangle=\frac{1}{2 \pi i} \oint \frac{d \zeta}{\zeta^{p+1}} \prod_{k}\left(u_{k}+v_{k} \zeta a_{k}^{\dagger} a_{k}^{\dagger}\right)|0\rangle
$$

where $\zeta=z^{2}$ and $p=N / 2$ is the number of particle pairs. The integrand in the above equation is a Laurent series in $\zeta$. Making use of the fermionic commutation relations for the operators $a_{k}, a_{k}^{\dagger}$, the matrix elements of an observable can be expressed by the residues

$$
R_{v}^{m}\left(k_{1}, \cdots, k_{m}\right)=\frac{1}{2 \pi i} \oint \frac{d z}{z^{(p-v)+1}} \prod_{k \neq k_{1}, \cdots, k_{m}>0}\left(u_{k}^{2}+z v_{k}^{2}\right)
$$

For example, the expectation value of energy is written as,

$$
\begin{aligned}
& E_{\text {proj }}^{A}=\frac{\left\langle\Psi^{A}|H| \Psi^{A}\right\rangle}{\left\langle\Psi^{A} \mid \Psi^{A}\right\rangle}=\left(R_{0}^{0}\right)^{-1}\left\{\sum_{k>0} \varepsilon_{k} v_{k}^{2} R_{1}^{1}(k)\right. \\
& \left.+\sum_{k, k^{\prime}>0}\left[G_{k k^{\prime} k k^{\prime}} v_{k}^{2} v_{k^{\prime}}^{2} R_{2}^{2}\left(k, k^{\prime}\right)+G_{k \bar{k} k^{\prime} \bar{k}^{\prime}} u_{k} v_{k} u_{k^{\prime}} v_{k^{\prime}} R_{1}^{2}\left(k, k^{\prime}\right)\right]\right\} .
\end{aligned}
$$

This projection method is termed as the PBCS theory.

Concurrence and discord. Consider a mixed state $\rho$ of two qubits $A$ and $B$. By introducing operator $\tilde{\rho}=\left(\sigma_{y} \otimes \sigma_{y}\right) \rho^{*}\left(\sigma_{y} \otimes \sigma_{y}\right)^{17}$, where $\sigma_{y}$ is the $y$ component of Pauli matrix, one defines the concurrence of $\rho$,

$$
\mathbb{C}(\rho)=\max \left\{0, \sqrt{\lambda_{1}}-\sqrt{\lambda_{2}}-\sqrt{\lambda_{3}}-\sqrt{\lambda_{4}}\right\},
$$

where $\lambda_{i}(i=1,2,3,4)$ are the eigenvalues of $\rho \tilde{\rho}$ in descending order. When $\mathbb{C}(\rho)>0$, qubits $A$ and $B$ are entangled.

Quantum discord, as another kind of correlation, may exist even without entanglement $^{3}$. The quantum correlation features itself with many aspects, e.g., in characterizing quantum phase transitions ${ }^{18}$. For the state $\rho$, quantum discord reads:

$$
\mathcal{D}(\rho)=\mathcal{I}(\rho)-\mathcal{C}(\rho),
$$

where $\mathcal{I}(\rho)$ is the quantum analogue of classical mutual information, defined as

$$
\mathcal{I}(\rho)=S\left(\rho^{A}\right)+S\left(\rho^{B}\right)-S(\rho),
$$

with $\rho^{A(B)}=\operatorname{Tr}_{B(A)}(\rho)$ denoting the reduced density matrix of the partition $A(B), S(\rho)$ the corresponding von Neumann entropy. $\mathcal{I}(\rho)$ is interpreted as a measure of total correlations in the composite system $A+B$, while $\mathcal{C}(\rho)$ is a measure of classical correlations, defined as

$$
\mathcal{C}(\rho)=S\left(\rho^{A}\right)-\sup _{\left\{B_{k}\right\}} S\left(\rho \mid\left\{B_{k}\right\}\right)
$$

where $\left\{B_{k}\right\}(k=0,1)$ stands for the von Neumann measurements on subsystem $B$. From the above definitions, we can see that quantum discord is the quantumness 
coming from quantum measurement, while entanglement is from the wave function (more generally density matrix) itself.

1. Bardeen, J., Cooper, L. N. \& Schriffer, R. S. Theory of Superconductivity. Phys. Rev. 108, 1175-1204 (1957).

2. Mastellone, A., Falci, G. \& Fazio, Rosario Small Superconducting Grain in the Canonical Ensemble. Phys. Rev. Lett. 80, 4542-4545 (1998).

3. Ollivier, H. \& Zurek, W. H. Quantum Discord: A Measure of the Quantumness of Correlations. Phys. Rev. Lett. 88, 017901 (2001).

4. Datta, A., Shaji, A. \& Caves, C. M. Quantum Discord and the Power of One Qubit Phys. Rev. Lett. 100, 050502 (2008).

5. Maziero, J., Celeri, L. C., Serra, R. M. \& Vedral, V. Classical and quantum correlations under decoherence. Phys. Rev. A 80, 044102 (2009).

6. Ferraro, A., Aolita, L., Cavalcanti, D., Cucchietti, F. M. \& Acín, A. Almost all quantum states have nonclassical correlations. Phys. Rev. A 81, 052318 (2010).

7. Bayman, B. F. A Derivation of the Pairing-correlation Method. Nucl. Phys. 15, 33 (1960) and references therein

8. Martiń-Delgado, M. A. Entanglement and Concurrence in the BCS State. arXiv:quant-ph/0207026v1.

9. Dusuel, S. \& Vidal, J. Finite-sizw scaling exponents and entanglement in the twolevel BCS model. Phys. Rev. A 71, 060304(R) (2005).

10. Ring, P. \& Schuck, P. The Nuclear Many-Body Problem. (Springer-Verlag, New York, 1980).

11. Ditrich, K., Mang, H. J. \& Pradal, J. H. Conservation of Particle Number in the Nuclear Pairing Model. Phys. Rev. 135, B22-B34 (1964).

12. Wu, L.-A., Byrd, M. S. \& Lidar, D. A. Polynomial-Time Simulation of Pairing Models on a Quantum Computer. Phys. Rev. Lett. 89, 057904 (2002).

13. Wu, L.-A. \& Lidar, D. A. Dressed Qubits. Phys. Rev. Lett. 91, 097904 (2003).

14. Wu, L.-A. \& Lidar, D. A. Qubits as parafermions. J. Math. Phys. 43, 4506-4525 (2002).

15. Wu, L.-A., Ding, H.-M. \& Jing, X.-G. Pairing correlations between the normal and abnormal parity orbits and the mechanism of the stretching effect. Phys. Rev. C52, 1845-1852 (1995)

16. Wu, L.-A., Zeng, G.-M. \& Jing, X.-G. Study on the $\gamma$-stability of nuclei in the light rare-earth region. Nucl. Phys. A 575, 85-92 (1994).

17. Wootters, W. K. Entanglement of Formation of an Arbitrary State of Two Qubits. Phys. Rev. Lett. 80, 2245-2248 (1998).

18. Sarandy, M. S. Classical correlation and quantum discord in critical systems. Phys. Rev. A 80, 022108 (2009).

19. Luo, S. Quantum discord for two-qubit systems. Phys. Rev. A 77, 042303 (2008)

20. Ali, M., Rau, A. R. P. \& Alber, G. Quantum discord for two-qubit X states. Phys. Rev. A 81, 042105-042111 (2010); Erratum: Quantum discord for two-qubit X states [Phys. Rev. A 81, 042105 (2010)]. Phys. Rev. A 82, 069902(E) (2010).

21. Chen, Q., Zhang, C., Yu, S., Yi, X. X. \& Oh, C. H. Quantum discord of two-qubit X states. Phys. Rev. A 84, 042313-042317 (2011).

22. Li, B., Wang, Z.-X. \& Fei, S.-M. Quantum discord and geometry for a class of twoqubit states. Phys. Rev. A 83, 022321 (2011).

23. Hu, M. L. \& Fan, H. Upper bound and shareability of quantum discord based on entropic uncertainty relations. Phys. Rev. A 88, 014105 (2013).
24. Yu, C. S., Jin, J. S., Fan, H. \& Song, H. S. Dual roles of quantum discord in a nondemolition probing task. Phys. Rev. A 87, 022113 (2013).

25. Mazzola, L., Piilo, J. \& Maniscalco, S. Sudden Transition between Classical and Quantum Decoherence. Phys. Rev. Lett. 104, 200401 (2010).

26. Maziero, J., Céri, L. C., Serra, R. M. \& Sarandy, M. S. Long-range quantum discord in critical spin systems. Phys. Lett. A 376, 1540-1544 (2012).

27. Groisman, B., Popescu, S. \& Winter, A. Quantum, classical, and total amount of correlations in a quantum state. Phys. Rev. A 72, 032317 (2005).

28. Henderson, L. \& Vedral, V. J. Phys. A 34, 6899 (2001); Vedral, V. Classical Correlations and Entanglement in Quantum Measurements. Phys. Rev. Lett. 90, 050401 (2003).

29. Horodecki, M., Horocecki, P., Horodecki, R., Oppenheim, J., Sen, A., Sen, U. \& Synak-Radtke, B. Local versus nonlocal information in quantum-information theory: Formalism and phenomena. Phys. Rev. A 71, 062307 (2005).

30. Wu, L.-A., Guidry, M., Sun, Y. \& Wu, C.-L. SO(5) as a critical dynamical symmetry in the SU(4) model of high-temperature superconductivity. Phys. Rev. B 67, 014515 (2003).

\section{Acknowledgments}

We are grateful to M. S. Sarandy for useful discussions. L.-A.W. acknowledges for financial support from the Basque Government (Grant No. IT472-10), the Spanish MICINN (Project No. FIS2012-36673-C03-03), and the Basque Country University UFI (Project No. 11/ 55-01-2013).

\section{Author contributions}

G.-M.Z. contributed to the theoretical derivations, numerical calculations and physical analysis, L.-A.W. to the conception and design of this work. Both authors participated in the discussions and the writing of all versions of the manuscript. H.-J.X. contributed to the initial derivation, calculation and analysis and participated in the first version of the manuscript

\section{Additional information}

Competing financial interests: The authors declare no competing financial interests.

How to cite this article: Zeng, G.-M., Wu, L.-A. \& Xing, H.-J. Symmetry restoration and quantumness reestablishment. Sci. Rep. 4, 6377; DOI:10.1038/srep06377 (2014).

This work is licensed under a Creative Commons Attribution-NonCommercialNoDerivs 4.0 International License. The images or other third party material in this article are included in the article's Creative Commons license, unless indicated otherwise in the credit line; if the material is not included under the Creative Commons license, users will need to obtain permission from the license holder in order to reproduce the material. To view a copy of this license, visit http:// creativecommons.org/licenses/by-nc-nd/4.0/ 